\title{
Contribution of thixotropy, spasticity, and contracture to ankle stiffness after stroke
}

\author{
Wantana Vattanasilp, Louise Ada, Jack Crosbie
}

\begin{abstract}
Objectives-Increased resistance to stretch of muscles after stroke may be the result of centrally mediated neural factors such as spasticity or local, peripheral factors such as muscle contracture or thixotropy. The aim was to investigate evidence for an abnormal thixotropic response and compare this with two other factorscontracture and spasticity-which could potentially contribute to muscle stiffness after stroke.

Methods-Thirty patients with stroke whose calf muscles were assessed clinically as stiff and 10 neurologically normal subjects were recruited. To measure thixotropy, their calf muscles were stretched through two cycles after two prestretch conditions: one in which the muscles were maintained in a shortened position and one in which they were maintained in a lengthened position. Spasticity was defined as the presence of tonic stretch reflexes in relaxed muscles. Contracture was defined as being present when maximum passive ankle dorsiflexion fell at least $2 \mathrm{SD}$ below the mean value of the control subjects.

Results-Both controls and patients with stroke exhibited a thixotropic response but this was no greater in the patients than the controls. About one third of the patients displayed muscle contracture and most exhibited spasticity. Contracture made a significant contribution $(p=0.006)$ to the clinical measure of calf muscle stiffness while spasticity made a significant contribution $(p=0.004)$ to the laboratory measure of calf muscle stiffness.
\end{abstract}

Conclusions-Measuring thixotropy at the level of joint movement was sufficiently sensitive to determine the thixotropic response in both neurologically normal subjects and patients impaired after stroke. The thixotropic response was not higher than normal after stroke, suggesting that whereas thixotropy may produce enough immediate resistance to impede movement in those who are very weak, it is not a substantial contributor to long term muscle stiffness. Contracture did significantly contribute to muscle stiffness, supporting the importance of prevention of contracture after stroke. Spasticity contributed to muscle stiffness only when the limb was moved quickly. (f Neurol Neurosurg Psychiatry 2000;69:34-39)

Keywords: thixotropy; muscle contracture; spasticity; stroke; calf muscles
Increased resistance to stretch-that is, stiffness, of the calf muscles after stroke-interferes with the function of the ankle joint. ${ }^{12}$ For example, stiff calf muscles may impede the performance of ankle dorsiflexion during the first phase of standing up and during the late stance phase in walking. Theoretically, increased resistance to passive movement can result from neural factors such as spasticity, or peripheral factors such as changes in the mechanical properties of the muscles (for example, as a result of muscle contracture). ${ }^{3}$

Increased resistance to passive movement after stroke is possibly a result of another phenomenon in muscle called "thixotropy". The term thixotropy has been applied to substances which can be changed from gel to solution after being stirred. Muscle behaves as a thixotropic substance in that its stiffness depends on the history of limb movement. ${ }^{4}$ For example, Lakie and Robson ${ }^{5}$ found that when stretch was applied to a relaxed muscle which had been maintained in a shortened position, the initial resistance was high, but if the muscle had been in a lengthened position the initial resistance was lower. Clinically, patients with stroke often display an increased resistance to passive movement when the paralysed limb has been sustained for a time with some muscles in a shortened position. After the paralysed muscles have been stretched, the resistance to movement decreases and the muscles become "looser". This clinical behaviour may be the result of an exaggerated thixotropic response as the thixotropic response in normal muscles is too small to notice. ${ }^{6}$

The aim of this study was to investigate factors contributing to muscle stiffness after stroke. Patients with stroke with stiff calf muscles were investigated to determine whether they displayed an abnormally high thixotropic response compared with able bodied subjects. Two other possible contributing factorscontracture and spasticity-were also examined.

\section{Methods}

SUBJECTS

Thirty patients with stroke were recruited to this study. They comprised 12 right and 18 left hemiplegic patients who were between 2 weeks and 5 years after their first stroke, with a mean age of 68 (SD 9) years. Patients were included if their calf muscles felt clinically stiff, had a score of increased muscle tone $\geqslant 2$ on the Ashworth scale, ${ }^{7}$ and had sufficient cognitive ability to participate in the procedures. They were excluded if they had sensory problems, musculoskeletal problems interfering with 
ankle joint movement, or any other neurological conditions. Ten neurologically normal subjects, mean age 59 (SD 8) years, acted as controls. The experimental procedures were approved by the relevant hospital and university ethics committees and informed consent was obtained before data collection commenced.

\section{MEASUREMENT OF THIXOTROPY}

To allow a thixotropic response to develop, the calf muscles were held in two different prestretch conditions for 5 minutes each: holdshort and hold-long. In the hold-short condition, the calf muscles were held in $10^{\circ}$ plantarflexion, whereas in the hold-long condition, the calf muscles were held in $10^{\circ}$ dorsiflexion. Calf muscles were stretched through two cycles after each prestretch condition.

Subjects were positioned in long sitting, with their leg and foot placed in a foot frame and secured by straps to prevent movement in the frame (fig 1). The frame employed in this study consisted of two rectangular perspex plates, one stationary and the other movable, articulated at a uniaxial hinge joint which contained a potentiometer calibrated to measure ankle displacement. A load cell was installed between the moveable plate and the handle to measure the force required to move the foot into dorsiflexion. The distance of the force to the axis was constant and the applied torque at the ankle was calculated as the product of the applied force and this distance. To check that the calf muscles were relaxed during the test, EMG was carried out using surface electrodes over the medial gastrocnemius muscle.

After 5 minutes in each prestretch condition, the ankle joint was stretched slowly backwards and forwards twice, between $10^{\circ}$ dorsiflexion and $10^{\circ}$ plantarflexion, at a velocity of $2 \%$ - that is, it took 40 seconds to complete the two stretching cycles. This very slow velocity minimised the likelihood of reflex activity

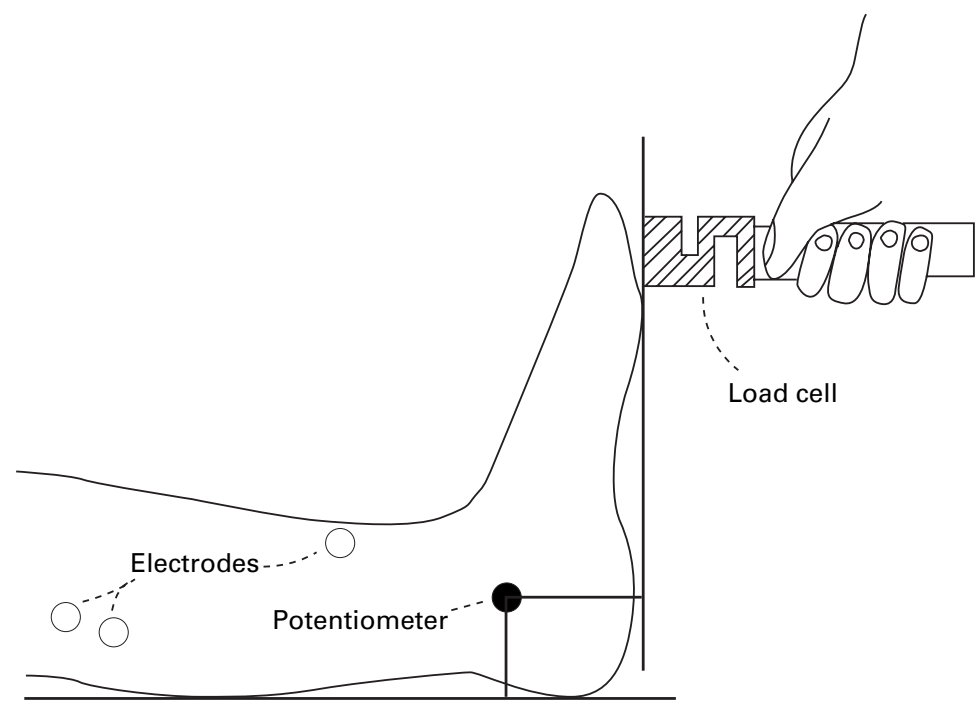

Figure 1-Diagram of the equipment used for measuring and recording of the EMG, ankle angle, and torque signals. The foot was positioned so that the lateral malleolus was aligned with the axis of rotation of the foot frame containing the potentiometer. The ankle joint could be rotated through different ranges at different speeds to measure thixotropy, contracture, and spasticity of the calf muscles.

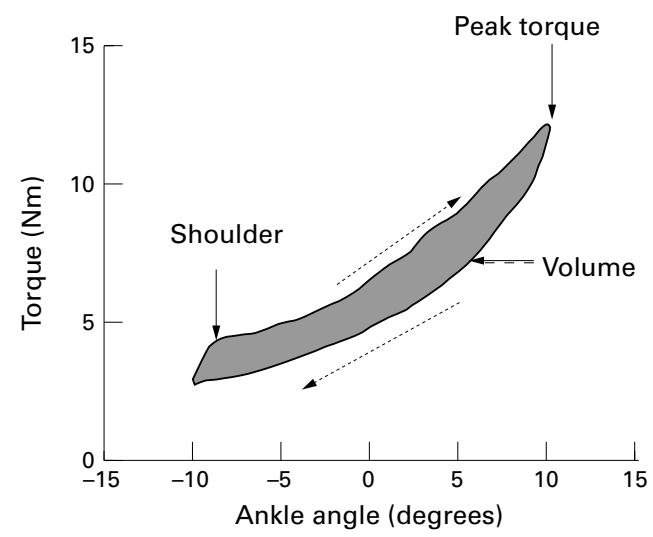

Figure 2 A torque angle curve of the first cycle of stretching after the hold-short condition from a patient with stroke. Dotted arrows indicate direction of stretch. The three variables used to describe the thixotropic

response — shoulder, volume, and peak torque — are indicated.

influencing the torque signal. Ankle angle was displayed on line as feedback so that the examiner could move the ankle joint smoothly within the specified range. Both ankle angle and torque signals were low pass filtered (digital, zero phase, 8th order Butterworth) at 1 $\mathrm{Hz}$. It is important to ensure that there is no neural activity around the joint when measuring thixotropy. A passive response is characterised by a smooth response. The ankle angle signal from each trial was double differentiated. The trial was discarded if $(a)$ there was an initial acceleration causing a high torque artefact or (b) there was a sudden acceleration as a result of voluntary activation of the muscles around the ankle joint. The torque angle curve of each stretching cycle was then analysed.

Three variables were quantified - the shoulder, volume, and torque of each torque angle curve to fully describe the history dependent changes to the calf muscles under the different conditions (fig 2). The shoulder represents the initial resistance to the stretch and was visually identified for each stretch cycle and its corresponding torque value recorded. The volume of the torque angle curve represents its area of hysteresis. The torque at $10^{\circ}$ dorsiflexion represents a general measure of overall stiffness. The magnitude of the thixotropic response was then quantified as the response of these three variables across stretching cycles as well as between stretching conditions. Thx-1 was defined as the difference in these variables between the 1 st and the 2 nd cycle of the holdshort condition - that is, it represents the short term thixotropic response. Thx-2 was defined as the difference in these variables between the 1 st cycle of the hold-short and hold-long conditions-that is, it represents the thixotropic response after a longer period of stretching, in this case 5 minutes.

MEASUREMENT OF CONTRACTURE

To investigate contracture, subjects were asked to relax their calf muscles while the examiner slowly dorsiflexed their ankle joint by pushing the movable plate of the foot frame until the 
heel just lifted off the movable plate. The lifting off of the heel constituted the operational definition of maximum passive ankle dorsiflexion. This definition was based on the fact that the calf muscles insert onto the posterior surface of the calcaneus via the Achilles tendon so they are most likely to significantly limit ankle dorsiflexion up to the point of heel lift off, with any further dorsiflexion probably coming from the movement of the forefoot on the talus. The EMG recording was displayed on line to check that the subject stayed relaxed. The ankle angle at heel lift off was then identified and recorded as maximum passive ankle dorsiflexion.

MEASUREMENT OF SPASTICITY

To investigate spasticity, the magnitude of abnormal reflex activity was measured. Subjects were asked to relax while the calf muscles were stretched sinusoidally. The examiner moved the foot backwards and forwards in the foot frame over a $20^{\circ}$ range between $10^{\circ}$ plantarflexion and $10^{\circ}$ dorsiflexion at $2 \mathrm{~Hz}$ for 30 seconds. The ankle angle was displayed online for feedback about displacement and a metronome was used for feedback about timing so that the examiner could accurately stretch the calf muscles within the specified range and frequency. The EMG was rectified and high pass filtered (digital, zero phase, 8th order Butterworth) at $80 \mathrm{~Hz}$ to remove any high frequency interference or movement artefact, then low pass filtered (digital, zero phase, 8th order Butterworth) at $4 \mathrm{~Hz}$ to obtain the IEMG signal.

The angle and IEMG were subjected to cross correlational and spectral analysis. This analysis allows activity at the stretching frequency $(2 \mathrm{~Hz})$ to be distinguished from activity unrelated to the stretch. Muscle activity that was time locked to the stretch with a phase lead was considered to be reflex in nature as it is not possible to voluntarily produce this pattern. ${ }^{8}$ The presence of this pattern of muscle activity was regarded as evidence of the existence of spasticity. The magnitude of spasticity is the amount of this reflex activity presented in $\mu \mathrm{V} /{ }^{\circ}$ (see Ada et $a l^{9}$ for more detail of this procedure).

MEASUREMENT OF STIFFNESS

Stiffness of the calf muscles was determined in two ways. Firstly, it was assessed clinically using the Ashworth scale. Secondly, it was quantified from the resistance measured via the load cell during the sinusoidal stretching procedure. Angle and torque were subjected to cross correlational and spectral analysis which allowed the resistance offered by the calf muscles at the stretching frequency $(2 \mathrm{~Hz})$ and stretching range $\left(10^{\circ}\right.$ plantarflexion to $10^{\circ}$ dorsiflexion) to be identified. The magnitude of stiffness measured in the laboratory is the angle torque relation at this frequency and range presented in $\mathrm{Nm} /{ }^{\circ}$.

\section{STATISTICAL ANALYSES}

For the measurement of thixotropy, each torque angle curve yielded three variables: shoulder, volume, and torque for two measures of thixotropy, Thx-1 and Thx-2. One way analyses of variance (ANOVAs) were used to compare the difference in these six variables between the patients with stroke and controls to ascertain whether stroke subjects exhibited an abnormal thixotropic response.

The measurement of maximum passive ankle dorsiflexion of the control subjects was normally distributed and therefore used to define the angle at which it was considered that patients with stroke had a contracture. To determine whether thixotropy, contracture, or spasticity were differentially contributing to calf muscle stiffness after stroke, multiple regression was used. A standard multiple regression was performed to analyse the contribution of the shoulder of Thx-2, maximum passive ankle dorsiflexion and reflex activity to calf muscle stiffness, measured both as Ashworth scale scores and as the torque angle relation during sinusoidal stretching. Thx-2 was used because this condition involved holding the calf muscles in a lengthened position for 5 minutes and would therefore be expected to produce the greatest thixotropic response.

\section{Results}

THIXOTROPY

The control subjects exhibited both a short term (Thx-1) and a long term (Thx-2) thixotropic response in their relaxed calf muscles (table). When Thx-1 was analysed, - that is, the difference between the 1 st and the 2nd cycles of the hold-short condition - the 1st cycle showed significantly greater responses than that of the 2 nd cycle in all three variables: shoulder $(\mathrm{F} 1,9=20.2, \quad \mathrm{p}=0.002)$, volume $(\mathrm{F} 1,9=28.4, \mathrm{p}<0.001)$, and torque $(\mathrm{F} 1,9=5.7$, $\mathrm{p}=0.04)$. There were also significant differences in the shoulder $(\mathrm{F} 1,9=22.1, \mathrm{p}=0.001)$, volume $(\mathrm{F} 1,9=36.8, \mathrm{p}<0.001)$, and the torque $(\mathrm{F} 1,9=26.2, \mathrm{p}<0.001)$ of the torque angle curves for Thx-2-that is, the difference

Thixotropic response for all subjects

\begin{tabular}{|c|c|c|c|c|c|c|c|c|}
\hline Subject & Condition & $\begin{array}{l}\text { Shoulder } \\
(\mathrm{Nm})\end{array}$ & $\begin{array}{l}\text { Volume } \\
\left(\mathrm{Nm}^{\circ}\right)\end{array}$ & $\begin{array}{l}\text { Peak torque } \\
(\mathrm{Nm})\end{array}$ & Thixotrophy & $\begin{array}{l}\text { Shoulder } \\
(\mathrm{Nm})\end{array}$ & $\begin{array}{l}\text { Volume } \\
\left(\mathrm{Nm} /{ }^{\circ}\right)\end{array}$ & $\operatorname{Peak}(\mathrm{Nm})$ \\
\hline \multirow[t]{3}{*}{ Control } & 1st cycle hold-short & $1.11(0.58)$ & $0.59(0.21)$ & $7.48(2.00)$ & & & & \\
\hline & 2nd cycle hold-short & $0.15(0.33)$ & $0.43(0.14)$ & $7.07(1.68)$ & Thx-1 & $0.96(0.67)$ & $0.16(0.10)$ & $0.40(0.53)$ \\
\hline & 1st cycle hold-long & $0.14(0.25)$ & $0.27(0.11)$ & $5.72(1.65)$ & Thx-2 & $0.97(0.65)$ & $0.32(0.17)$ & $1.76(1.09)$ \\
\hline \multirow[t]{3}{*}{ All stroke } & 1st cycle hold-short & $0.63(0.52)$ & $0.53(0.15)$ & $7.88(2.12)$ & & & & \\
\hline & 2nd cycle hold-short & $0.09(0.24)$ & $0.36(0.10)$ & $7.35(2.16)$ & Thx-1 & $0.54(0.50)$ & $0.17(0.10)$ & $0.53(0.52)$ \\
\hline & 1st cycle hold-long & $0.07(0.19)$ & $0.30(0.09)$ & $5.59(1.54)$ & Thx-2 & $0.56(0.48)$ & $0.23(0.14)$ & $2.29(1.32)$ \\
\hline \multirow[t]{3}{*}{ Stroke with contracture } & 1st cycle hold-short & $0.64(0.52)$ & $0.55(0.21)$ & $9.02(2.04)$ & & & & \\
\hline & 2nd cycle hold-short & $0.05(0.17)$ & $0.35(0.11)$ & $8.26(2.24)$ & Thx-1 & $0.59(0.56)$ & $0.20(0.13)$ & $0.40(0.16)$ \\
\hline & 1 st cycle nold-long & $0.00(0.00)$ & $0.32(0.10)$ & $6.30(1.72)$ & Thx-2 & $0.64(0.52)$ & $0.23(0.20)$ & $2.72(1.71)$ \\
\hline
\end{tabular}


between the 1st cycle of the hold-short and that of the hold-long conditions. In other words, after holding the relaxed muscle in a shortened position, there was a greater resistance to movement compared with that after holding the muscle in a lengthened position whether the lengthened position represented just one cycle of stretching or 5 minutes of stretching.

Patients with stroke also exhibited both a short term (Thx-1) and a long term (Thx-2) thixotropic response in their relaxed calf muscles (table). When Thx-1 was analysed, the 1 st cycle showed significantly greater responses than that of the 2 nd cycle in all three variables: the shoulder $(\mathrm{F} 1,29=34.9, \mathrm{p}<0.001)$, volume $(\mathrm{F} 1,29=80, \quad \mathrm{p}<0.001), \quad$ and torque $(\mathrm{F} 1,29=31.2, \mathrm{p}<0.001)$. There were also significant differences in the shoulder $(\mathrm{F} 1,29=40.5, \mathrm{p}<0.001)$, volume $(\mathrm{F} 1,29=76$, $\mathrm{p}<0.001)$ and the torque $(\mathrm{F} 1,29=91.2$, $\mathrm{p}<0.001$ ) of the torque angle curves for Thx-2.

When the magnitude of both thixotropic responses, Thx-1 and Thx- 2 were compared between the patients with stroke and control subjects (table), there were no significant differences in two of the variables: the volume $(\mathrm{F} 1,38=0.01, \mathrm{p}=0.92$ for $\mathrm{Thx}-1$ and $\mathrm{F} 1,38=2.86, \mathrm{p}=0.10$ for $\mathrm{Thx}-2)$ and the torque $(\mathrm{F} 1,38=0.44, \mathrm{p}=0.51$ for $\mathrm{Thx}-1$ and $\mathrm{F} 1,38=1.34, \mathrm{p}=0.25$ for $\mathrm{Thx}-2$ ) between both groups (fig $3 \mathrm{~A}$ and $\mathrm{B}$ ). Only the difference in the change of the shoulder of the torque angle curve was significantly different between the patients with stroke and control subjects ( $\mathrm{F} 1,38=4.29, \quad \mathrm{p}=0.05$ for $\mathrm{Thx}-1$ and $\mathrm{F} 1,38=4.54, \mathrm{p}=0.04$ for $\mathrm{Thx}-2)$. However, the change in the shoulder of the torque angle curve in the controls was greater than that in patients with stroke $(0.96$ (SD 0.67) $\mathrm{Nm} v$

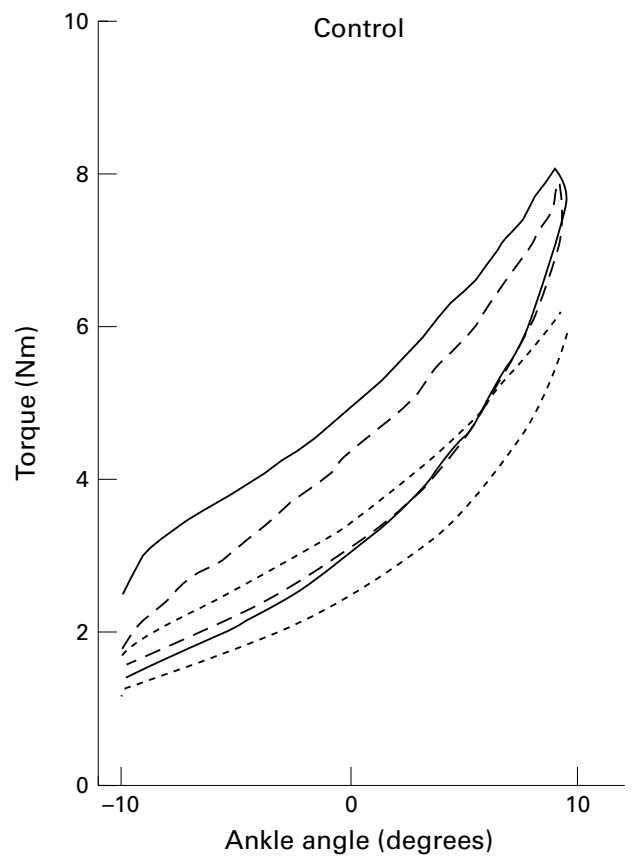

0.54 (SD 0.50) Nm for Thx-1 and 0.97 (SD $0.65) \mathrm{Nm} v 0.56$ (SD 0.48) Nm for Thx-2) illustrating a greater thixotropic response normally.

\section{CONTRACTURE}

Patients with stroke in this study displayed significantly less maximum passive ankle dorsiflexion $\left(9.2(\mathrm{SD} \mathrm{3.1})^{\circ}\right)$ compared with control subjects $\left(15.3 \quad(\mathrm{SD} 3.5)^{\circ}\right) \quad(\mathrm{F} 1,38=26.9$, $\mathrm{p}<0.001)$. For this study, patients who displayed a maximum passive ankle dorsiflexion value of less than $2 \mathrm{SD}$ from the mean value of the control subjects were considered as having a calf muscle contracture. According to this definition, 11 of the 30 patients had contracture-that is, their maximum passive ankle dorsiflexion was less than $8.26^{\circ}$, and 19 patients had no contracture.

\section{SPASTICITY}

When the relaxed gastrocnemius was stretched, none of the control subjects exhibited reflex activity - that is, they were not spastic. By contrast, 25 of the 30 patients exhibited spasticity $(83 \%)$. However, the magnitude of the reflex activity was small (0.20 (SD 0.21) $\left.\mu \mathrm{V} /{ }^{\circ}\right)$.

CONTRIBUTION OF THIXOTROPY, CONTRACTURE, AND SPASTICITY TO CALF MUSCLE STIFFNESS A standard multiple regression was performed to analyse the contribution of shoulder from Thx-2, maximum passive ankle dorsiflexion and reflex activity to calf muscle stiffness, measured both as Ashworth scale scores and as the torque angle relation during sinusoidal stretching. The results of the first analysis showed that only the variable maximum

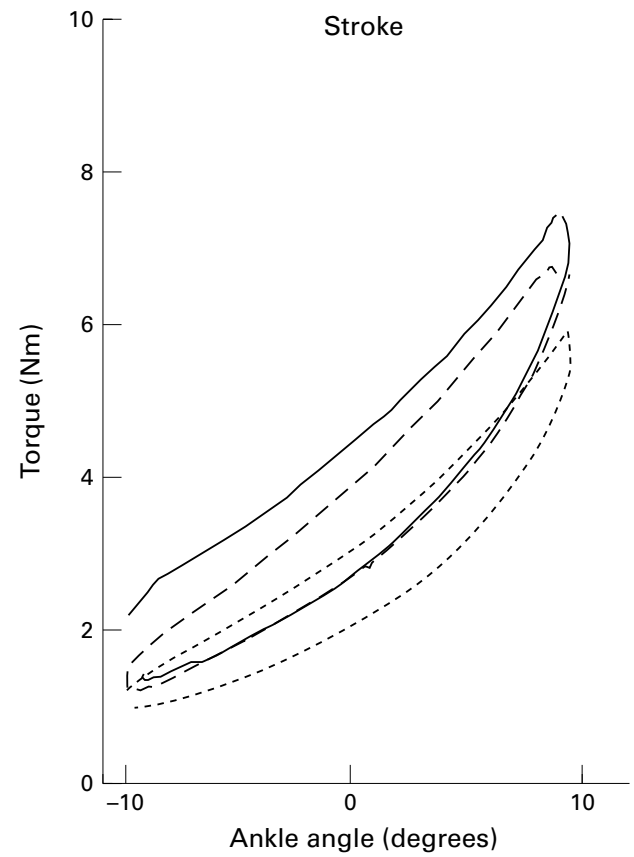

Figure 3 Mean torque angle curves of (a) the first cycle of stretching after the hold-short condition (solid line), (b) the second cycle of stretching after the hold-short condition (dashed line), and (c) the first cycle of stretching after the hold-long condition (dotted line), in control (left graph) compared with patients with stroke (right graph). The difference in the shoulder, volume, and torque of the first and second torque angle curves represents the short term thixotropic response $(T h x-1)$ whereas the difference in the shoulder, volume, and torque of the first and third torque angle curves represents the long term thixotropic response (Thx-2). 
passive ankle dorsiflexion contributed significantly $(\mathrm{F} 1,26=8.9, \mathrm{p}=0.006)$ to calf muscle stiffness as quantified by the Ashworth scale scores, accounting for $25 \%$ of the variance. The results of the second analysis showed that only the variable reflex activity contributed significantly $(\mathrm{F} 1,26=10.0, \mathrm{p}=0.004)$ to calf muscle stiffness as quantified by the torque angle relation during sinusoidal stretching, accounting for $27 \%$ of the variance.

\section{Discussion}

The aim of this study was to investigate evidence of an abnormal thixotropic response, and other possible factors contributing to stiffness, in patients with stroke whose calf muscles felt clinically stiff. The thixotropic response of normal subjects was examined and used as a reference for comparison with patients. Thixotropy was measured at the level of joint movement in this study. Although this does not allow for elaboration of underlying mechanisms such as those put forward by Hill ${ }^{10}$ and Campbell and Lakie, ${ }^{11}$ the application of slow displacements to the ankle joint and measurement of the resultant torque after two different prestretch conditions was found to be sufficiently sensitive to detect the thixotropic response. Neurologically normal subjects exhibited both a short term and long term thixotropic response. That is, three variables of the torque angle curve analysed-the shoulder (initial resistance), volume and torque-were significantly greater in the 1 st cycle of the hold-short condition compared with that in the 2nd cycle of the same condition as well as the 1 st cycle of the hold-long condition.

A thixotropic response was also found in the relaxed calf muscles of patients with stroke. This finding was in line with previous studies that thixotropy appeared in pathological conditions such as in the fingers of children with cerebral palsy ${ }^{12}$ and in the knee joint of a tetraplegic patient. ${ }^{13}$ The patients with stroke did not seem to exhibit an abnormally high response compared with the controls. Changes in two of the three variables were not significantly different between normal subjects and patients. Only the change in the shoulder of the torque angle curve was significantly different. It is important to note, however, that the thixotropic response was greater in the controls than the patients with stroke, the opposite of what would be expected if the patients with stroke had an abnormally high thixotropic response. Of course, the very slow time course of the stretch of the calf muscles may have underestimated the thixotropic response, which may be larger if the muscle is either left for longer to develop thixotropy or stretched faster after having developed thixotropy. Even though the patients with stroke did not seem to exhibit an abnormal thixotropic response, the presence of thixotropy may offer sufficient resistance that patients with stroke who are very weak may not be able to initiate movement after a period of immobility - for example, weak wrist extensors may not be able to overcome the thixotropic resistance of wrist flexors which have been resting in a shortened position for some time.
Furthermore, the finding that the patients with stroke exhibited a short term thixotropic response- that is, a reduction in stiffness after only one passive stretching cycle-implies that stretching muscles for a few seconds, performed by the patients themselves or a therapist, can reduce resistance to movement.

About one third of patients with stroke showed muscle contracture whereas most exhibited spasticity in their calf muscles. Multiple regression analysis disclosed that spasticity was making a significant contribution to the stiffness of the calf muscles when assessed in the laboratory. This finding is in line with a previous study which has shown that spasticity can contribute to calf muscle stiffness when measured as the torque angle relation during sinusoidal stretching ${ }^{9}$ and is consistent with Lance's definition of spasticity which implies that hyperreflexia can contribute to an increased resistance to movement. ${ }^{14}$ On the other hand, multiple regression analysis showed that contracture was the only variable making a significant contribution to the stiffness of the calf muscles when assessed clinically. This finding is in line with a previous study which has suggested that the increased resistance to passive movement was not dependent on the presence of spasticity, and that contracture alone can play an important part in contributing to stiffness after stroke. ${ }^{15}$

The findings presented here support the conclusions from previous studies that the Ashworth scale cannot identify the cause of resistance ${ }^{1617}$ because it measures both the neural and peripheral contributions without differentiating their individual contributions. The different results of the two analyses may be the result of the difference in speed of movement during the two tests of calf muscle stiffness. During grading using the Ashworth scale, the ankle is moved relatively slowly. As the reflex activity is velocity dependent, this movement may be too slow to excite abnormal reflex activity whereas movement during the sinusoidal stretching was much faster. Previously we have shown very little reflex activity present in the calf muscles of patients with stroke when stretching was applied at $0.5 \mathrm{~Hz}$ compared with $2 \mathrm{~Hz}$ as in the present study. ${ }^{9}$ Therefore, when the Ashworth scale is used as a measure of spasticity, it can overestimate spasticity because it assumes that any increase in resistance felt is the result of abnormal reflex activity. However, in this group of patients with stroke, the small amount of spasticity in the calf muscles made only a minor contribution to the increased resistance recorded as Ashworth scores ranging from 2 to 4 , compared with contracture. It is important, therefore, that the Ashworth scale is used as a clinical measure of stiffness (or hypertonia) rather than of spasticity. The Tardieu scale may be better at identifying a neural component to stiffness because the test involves moving the limb at different velocities and comparing the difference in the response. The Tardieu scale has been little known because it was originally published only in French ${ }^{18}$ but Boyd et al have recently had the scale translated into English. ${ }^{19}$ 
The question of an interaction between the two peripheral factors, thixotropy and contracture, was investigated. A post hoc test was applied to investigate the magnitude of the thixotropic response in the patients with stroke who had contracture. However, when both short term and long term thixotropic responses were examined, patients with stroke with contracture had no greater response in any aspect of their torque angle curves than those without contracture. This suggests that the thixotropic response is not made substantially worse by the presence of contracture.

In conclusion, this study investigated three possible factors, thixotropy, contracture, and spasticity, which could contribute to passive stiffness of the ankle joint in patients with stroke who were assessed as clinically stiff. It was found that the patients with stroke exhibited thixotropy, but that it was no greater than normal. The finding that contracture significantly increased the resistance of the ankle joint to passive movement when stretched at speeds used in the clinic adds to the growing body of evidence that suggests that peripheral factors are important contributors to muscle stiffness. $^{12}{ }^{20-23}$ This finding also supports previous suggestions that the need to prevent muscle contracture after stroke is vital..$^{1524}{ }^{25} \mathrm{As}$ contracture is much easier to prevent than to treat, ${ }^{26}$ paralysed muscles should not be left in a shortened range during prolonged periods of immobility after stroke. We are grateful to the physiotherapy staff and patients of
Bankstown-Lidcombe, Liverpool, St George, and Royal Prince Bankstown-Lidcombe, Liverpool, St George, and Royal Prince
Alfred Hospitals for their assistance in this study. This work was supported by a University of Sydney research grant.

1 Sinkjær T, Magnussen I. Passive intrinsic and reflexmediated stiffness in the ankle extensors of hemiparetic mediated stiffness in the ankle
patients. Brain 1994;117:355-63.

2 Thilmann AF, Fellows SJ, Ross HF. Biomechanical changes at the ankle joint after stroke. F Neurol Neurosurg Psychiatry at the ankle join

3 Katz RT, Rymer WZ. Spastic hypertonia: mechanisms and measurement. Arch Phys Med Rehabil 1989;70:144-55.

4 Hagbarth K-E, Hagglund JV, Nordin M, et al. Thixotropic behavior of human finger flexor muscles with accompany- ing changes in spindle and reflex responses to stretch. 7 Physiol 1985;368:323-42.

5 Lakie M, Robson LG. Thixotropic changes in human muscle stiffness and the effects of fatigue. Q尹 Exp Physiol 1988; 73:487-500

6 Hufschmidt A, Schwaller I. Short-range elasticity and resting tension of relaxed human lower leg muscles. $f$ Physiol 1987;391:451-65.

7 Ashworth B. Preliminary trial of carisoprodal in multiple sclerosis. Practitioner 1964;192:540-2.

8 Neilson PD. Speed of response or bandwidth of voluntary system controlling elbow position in intact man. Medical and Biological Engineering 1972;10:450-9.

9 Ada L, Vattanasilp W, O'Dwyer NJ, et al. Does spasticity contribute to walking dysfunction after stroke?. F Neurol contribute to walking dysfunction after

10 Hill DK. Tension due to interaction between the sliding filaments in resting striated muscle. The effect of stimulation. $\mathcal{F}$ Physiol 1968;199:637-84

11 Campbell KS, Lakie M. A cross-bridge mechanism can explain the thixotropic short-range elastic component of relaxed frog skeletal muscle. F Physiol 1998;510:941-62.

12 Brown JK, Rensburg FV, Walsh G, et al. A neurologica study of hand function of hemiplegic children. Dev Med Child Neurol 1987;29:287-304.

13 Walsh EG. Postural thixotropy: a significant factor in the stiffness of paralysed limbs? Paraplegia 1992;30:113-5.

14 Lance JW. Symposium synopsis. In: Young RR, Feldman RG, Koella WP, eds. Spasticity: disordered motor control. RG, Koella WP, eds. Spasticity: disordered

15 O'Dwyer NJ, Ada L, Neilson PD. Spasticity and muscle contracture following stroke. Brain 1996;119:1737-49.

16 Nielsen JF, Sinkjær T. A comparison of clinical and laboratory measures of spasticity. Multiple Sclerosis 1996;1:296301 .

17 Vattanasilp W, Ada L. The relationship between clinical and aboratory measures of spasticity. Australian fournal of Physiotherapy 1999;45:135-9.

18 Tardieu G, Shentoub S, Delarue R. A la recherche d'une technique de measure de la spasticite. Rev Neurol 1954;91: 143-4.

19 Boyd RN, Barwood SA, Ballieu C, et al. Validity of a clinical measure of spasticity in children with cerebral palsy in a double blinded randomised controlled clinical trial. Dev Med Child Neurol 1998;40(suppl 78):7.

20 Dietz V, Quintern J, Berger W. Electrophysiological studies of gait in spasticity and rigidity. Brain 1981;104:431-49.

21 Hufschmidt A, Mauritz KH. Chronic transformation of muscle in spasticity. A peripheral contribution to increased tone. $\mathcal{F}$ Neurol Neurosurg Psychiatry 1985;48:676-85.

22 Ibrahim IK, Berger W, Trippel M, et al. Stretch-induced electromyographic activity and torque in spastic elbow muscles. Differential modulation of reflex activity in passive and active motor tasks. Brain 1993;116:971-89.

23 Carey JR, Burghardt TP. Movement dysfunction following central nervous system lesions: a problem of neurologic or muscular impairment? Phys Ther 1993; 73:538-47.

24 Carr JH, Shepherd RB, Ada L. Spasticity: research findings and implications for intervention. Physiotherapy 1995;81: 421-9.

25 Perry J. Rehabilitation of spasticity. In: Feldman RG, Young RR, Koella WP, eds. Spasticity: disordered motor control. Miami: Symposia Specialists, 1980:87-100.

26 Botte MJ, Nickel VL, Akeson WH. Spasticity and contracture. Physiologic aspects of formation. Clin Orthop 1988;233:7-18 